\title{
Subaortic stenosis caused by an unusual fibrous blood-filled cyst of the left ventricle with outflow tract obstruction associated with a ventricular septal defect
}

Seimei Nakae, Atsushi Kurata, Akira Ishihara

\begin{abstract}
A large blood-filled cyst formed from a fibrous tissue tag of a right ventricular septal aneurysm was successfully resected. This cyst, which was causing subaortic stenosis, was attached to the margin of the closed ventricular septal defect and not to the mitral valve itself nor the papillary muscle of the left ventricle.
\end{abstract}

Left ventricular outflow tract obstruction is often associated with anomalies of the mitral valve apparatus. It almost always originates from the papillary muscle or the mitral valve itself. ${ }^{12}$ We report a patient in whom the obstructive lesion was an unusual blood-filled cyst formed from a fibrous tissue tag of right ventricular septal aneurysm. This case reminded us that anomalies of both the right and left atrioventricular valve tissue anomalies can cause obstructive lesions. ${ }^{3}$

\section{Case report}

The patient weighed $3350 \mathrm{~g}$ at birth after an uneventful delivery. When he was three months old a systolic murmur and dextrocardia were noted. Echocardiography performed then and a year and a half later showed a perimembranous ventricular septal defect. The second echocardiographic examination also showed anomalous membranous tissue attached to the membranous portion of the left ventricle. Cross sectional echocardiography performed when the patient was seven years old showed that the ventricular septal defect was closed by a septal aneurysm, and a balloon shaped mass that was $18 \mathrm{~mm}$ in diameter with an echolucent centre in the left ventricular outflow tract. Cardiac catheterisation and left ventriculography were performed. Angiography showed that the large cyst moved to the subaortic portion during systole and a pressure gradient of $65 \mathrm{~mm} \mathrm{Hg}$ across the cyst (fig 1). An electrocardiogram showed sinus rhythm, an axis of $+100 \%$, and evidence of left ventricular hypertrophy. Echocardiographic examination showed a wall thickness of $7 \mathrm{~mm}$. The chest $x$ ray showed a cardiothoracic ratio of $52 \%$. At operation right ventriculotomy showed a ventricular septal aneurysm. The septal aneurysm communicated with the left ventricle via a small orifice. The communication was closed after the membranous septal aneurysm was resected. Subsequent aortotomy showed a large smooth, white, round fibrous cyst occupying the entire diameter of the left ventricular outflow tract and attached to the tissue between the perimembranous portion and ventricular septum, which was partly connected to the fibrous tissue that communicated with the ventricular septal aneurysm. It was thought that this fibrous cyst was associated with the septal aneurysm of the right ventricle and dependent on the mitral apparatus (fig 2). This round fibrous cyst (20 $\mathrm{mm}$ in diameter) was filled with blood. During the resection the blood escaped through a small orifice. The postoperative course was smooth and postoperative cardiac catheterisation showed neither a ventricular communication nor a pressure gradient from the left ventricular outflow tract to the ventricular apex. Macroscopic examination confirmed that the cyst was composed of white, elastic fibrous and gelatinous tissue. Histological examination showed that the cyst wall was made of mucus and degenerated fibrous tissue but not cardiac muscle fibre; these components were identical to those in the ventricular septal aneurysm resected from the right ventricle.



Figure 1 Left ventriculogram showing a large bloodfilled cyst in the subaortic portion of the left ventricular outflow tract. BC, blood-filled cyst. 


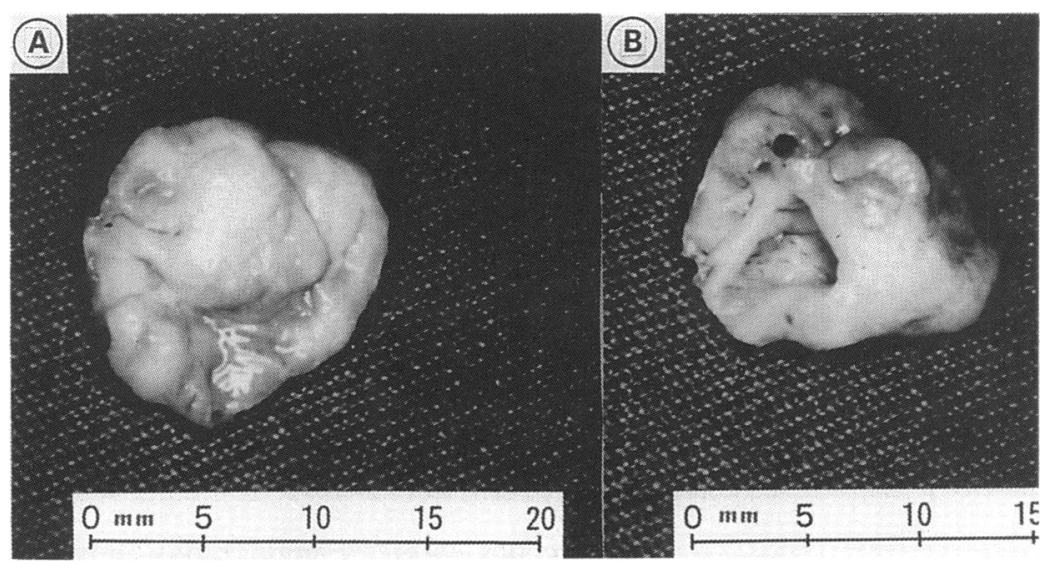

Figure 2 Cyst after removal through the aorta. (A) It had a small orifice at the apical side of the ventricle. (B) The aortic view shows the smooth surface. The wall was $2.0 \mathrm{~mm}$ thick. tricular septal defect during the course of closure of the ventricular septal defect. ${ }^{3}$ The small posterior orifice in the cyst accords with the hypothesis that the cyst became enlarged by filling up with blood under systolic pressure. ${ }^{4}$ The cyst became spherical as blood became trapped in its cavity. However, this blood was discharged through an orifice in the cyst after it was removed. Andersen proposed that maturational closure of a perimembranous ventricular septal defect and obstruction of the left ventricular outflow tract are the mechanisms by which a fibrous diaphragm, fibrous muscular tunnel, anomalous insertion of the atrioventricular valve, or aneurysmal fibrous tag develop. ${ }^{5}$ A fibrous tag could have protruded into the left ventricular cavity when the right ventricular pressure rose. Cross sectional echocardiography is essential to confirm that this type of blood-filled cyst showing unusual echolucent mass ${ }^{6}$ and attachment can be resected without mitral valve replacement.

There are several reports of obstruction of the left ventricular outflow tract by a blood-filled cyst attached to the mitral apparatus, by a bleb on the mitral valve, or by accessory tissue from the mitral valve..$^{-4}$ In our patient, however, the cyst was neither a simple bloodfilled cyst that had entrapped blood in the mitral valve apparatus nor a mitral bleb that obstructed the left ventricular outflow tract. Investigation during surgery confirmed that there was no connection between the fibrous cyst and either the mitral valve leaflets or the chordae tendineae of the mitral valve. This fibrous blood-filled cyst seemed to have originated and grown from the fibrous tissue tag of an aneurysm of the ventricular septum or from the accessory tissue of the tricuspid valve that had herniated through the ven-
We thank Dr S Hiraishi and Dr Y Agata for interpreting the echocardiograms.

1 Hauser AM, Rathod L, McGill J, Rosenberg BF, Gordon S, Timmis GC. Blood cyst of the papillary muscle. $A m$ Cardiol 1983;51:612-3.

2 Leatherman L, Leachman RD, Hallman GL, Cooley DA Cyst of mitral valve. Am J Cardiol 1968;21:428-30.

3 Sellers RD, Lillehei CW, Edwards JE. Subaortic stenosi caused by anomalies of atrioventricular valves. $J$ Thorac Cardiovasc Surg 1964;48:289-302.

4 MacLean LD, Culligan JA, Kane DJ. Subaortic stenosis due to accessory tissue on the mitral valve. $J$ Thorac Cardiovas

5 Anderson RH, Lennox CC, Zuberbuhler JR. Mechanisms of closure of perimembranous ventricular septal defect. Am J Cardiol 1983;52:341-5.

6 Arnold I, Hubner PJB, Firmin RK. Blood filled cyst of the papillary muscle of the mitral valve producing severe left ventricular outflow tract obstruction. Br Heart J 1990; 63:132-3. Surg 1963;45:382-8. 